\title{
Análise de Materiais Educativos Utilizados nas Campanhas de Saúde Coletiva Sobre o Tabagismo
}

\section{Analysis of Educational Materials Used in Public Health Campaigns on Smoking}

\author{
Antonio Sales*ab; Mariana Peres Rodrigues ${ }^{\mathrm{b}}$; Matheus Marques da Silva Santos ; Giovana Marcelino Stilben de Souzac; \\ Leda Marcia Araujo Bento ${ }^{b}$ \\ anniversidade Estadual de Mato Grosso do Sul. MS, Brasil.

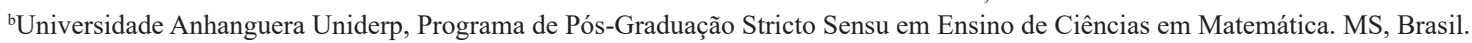 \\ 'Universidade Anhanguera Uniderp, Curso de Medicina. MS, Brasil. \\ *E-mail: profesales@hotmail.com
}

\begin{abstract}
Resumo
Este artigo é resultado de uma pesquisa documental tendo como base a análise de um material educativo elaborado com a finalidade de conscientizar a população sobre os malefícios do tabagismo. O documento analisado foi retirado do site da Fundação Portuguesa de Cardiologia em consideração ao dia nacional do não fumador. Contém uma revisão bibliográfica sobre os inconvenientes do tabaco, os males que acarreta e as substâncias nocivas que apresenta. Como referencial teórico foi utilizada a tipologia de conteúdos de ensino proposta por Antoni Zabala e a análise considerou os tipos de conteúdo presentes no material utilizado na referida campanha. Após o estudo concluiu-se que o material aborda vários tipos de conteúdos, podendo assim, contribuir para a sensibilização da população-alvo tendo potencial para induzir a uma atitude de mudança além de atingir a população não fumante como modo de prevenção em saúde.
\end{abstract}

Palavras-chave: Materiais Educativos. Campanhas de Saúde. Educação em Saúde.

\begin{abstract}
This article is the documentary research's result based on an educational material elaborated with the aim of making the population aware about the damages from smoking. The analyzed document was taken from the site of Cardiology's Portuguese Foundation in consideration of the non-smoker national's day. It contains a bibliographic review about the inconveniences of tobacco, the illness that it entails and the harmful substances it presents. The typology of teaching contents proposed by Antoni Zabala was used as a theoretical reference and the analysis considered the types of contents present in the material applied in that campain. After the research it was concluded that the material addresses several types of contents, thus contributing to the vulnerability of smoking population with te potential to induce a change's attitude besides affecting the non-smoker population as a way of health's prevention.
\end{abstract}

Keywords: Educational Materials. Health Campaigns. Health Education.

\section{Introdução}

O tabagismo é um costume praticado por adultos e também por inúmeros jovens mesmo sendo vedado a venda para menores de 18 anos. Essa prática é abundantemente nociva para saúde, pois ocasiona diversos danos físicos que podem levar à morte, danos psicológicos e comportamentais, além de afetar também fumantes passivos. Em virtude disso vem sendo combatido por meio de campanhas educativas promovidas pelo Ministério da Saúde, Secretarias Estaduais de Saúde, por Organizações Não Governamentais - ONG e movimentos religiosos. Dessa forma, pressupõe-se que se torna cada vez mais relevantes as campanhas educativas visando a mudança de atitude por parte da população.

Conforme o Instituto Nacional de Câncer (INCA, 2016) o cigarro contém 4.700 substâncias tóxicas ao organismo podendo acarretar diversos danos à saúde como o câncer de pulmão, de orofaringe, bexiga, laringe, colo de útero, além de doenças cardiovasculares. Uma dessas substâncias é a nicotina, que causa o vício nos fumantes, de acordo com Marques et al. (2001), essa dependência é decorrente da sua ação no sistema nervoso fazendo com que a pessoa sinta sensações agradáveis como de calma e relaxamento. Entretanto, quando o efeito cessa, a pessoa sente novamente a necessidade de usar cigarro.

O tabagismo causa efeitos tanto físicos quanto psicológicos e comportamentais. Esses efeitos são danosos à saúde merecendo, portanto, que não se poupem esforços para que o seu uso seja evitado. O cigarro é uma droga lícita no Brasil e conforme a legislação deveria ser vendido somente para maiores de 18 anos, entretanto, esta lei nem sempre é cumprida. Em virtude, da ausência de fiscalização efetiva uma das alternativas é a conscientização por meio de campanhas educativas. É importante salientar também, que os malefícios à saúde causados pelo tabagismo não afetam somente o fumante ativo, mas também o fumante passivo pela inalação da fumaça. Dentre os efeitos facilmente percebidos estão a cefaleia, alergias, irritação nos olhos e a tosse que importuna, além do odor não agradável que o ambiente conserva após a prática de fumar (BRASIL, 2011)

Existe cerca de 1,3 bilhões de fumantes no mundo, dos quais 800 milhões concentram-se em países em desenvolvimento. Dados de 2014 mostram que das cinco 
milhões de mortes por doenças causadas pelo tabaco, 50\% ocorrem em países em desenvolvimento (SALEHEEN; ZHAO; RASHEED, 2014). Evidenciando a importância da ação antitabagismo para jovens com materiais educativos contendo uma linguagem clara, objetiva, dados e imagens marcantes para que possa alcançar o objetivo esperado. Ou seja, conscientizar os fumantes sobre os males do tabagismo e os que ainda não possuem o hábito, que não iniciem. Sendo assim, haverá redução na mortalidade e comorbidades decorrentes do tabagismo resultando no aumento da qualidade e de expectativa de vida da população em vários aspectos: saúde física, econômica, mental e comportamental.

A pesquisa partiu do pressuposto de que a transformação do comportamento é fundamental e se propôs analisar e descrever os tipos de conteúdo presentes no material educativo (INCA, 2016) em consideração ao dia nacional do não fumador e articular esses conteúdos com os objetivos da campanha.

\section{Desenvolvimento}

\subsection{Metodologia}

A pesquisa se caracteriza como qualitativa documental. Cellard (2008) expõe que ao realizar uma análise documental é intrínseco tomar certos cuidados ao colocar a identificação de textos pertinentes e avaliar sua credibilidade. O pesquisador também deve compreender o sentido da mensagem, que está sendo veiculada, adequadamente. Ademais, difere os documentos em públicos, privados e pessoais, sendo o primeiro, uma documentação volumosa, variável no tempo e, em inúmeros momentos, não são acessíveis, apesar de serem públicos. Um exemplo desses arquivos são os documentos federais, regionais. Os documentos privados são caracterizados por serem arquivos que não pertença ao público, mas sim de instituições como empresas e igrejas. Por fim, os arquivos pessoais, que são correspondências, documentos familiares e histórias de vida.

Para efeitos de pesquisa, de análise ou investigação, distinguimos os documentos públicos em duas categorias: os que são totalmente públicos. Isto é, aqueles que tendo a sua origem no poder público são de livre acesso à população, como sites e materiais de campanha, por exemplo. Na outra categoria estão os documentos de origem em órgão público, porém, no que se refere ao acesso a eles pode-se dizer são apenas potencialmente públicos, isto é, o acesso é limitado necessitando-se para isso do cumprimento de determinado ritual burocrático.

A pesquisa consistiu na análise de um documento totalmente público. Tem origem no poder público e o acesso a ele é inteiramente livre e incentivado.

Segundo Martins (2004), os métodos classificados como qualitativos são metodologias que visam a análise do objeto de estudo. Para que isso ocorra, o pesquisador necessita compreender muito bem o texto que está sendo lido para realizar uma boa análise. Ainda, apresenta características para um método qualitativo sendo a flexibilidade quanto à melhor maneira de efetivar a coleta dos dados.

Para realização da análise qualitativa, é fundamental salientar que o principal verbo que comandou essa análise foi o verbo compreender. Para Minayo (2012) esse verbo essencial é a capacidade de empatia, ou seja, se colocar no lugar do outro, entendendo melhor a mensagem que o objeto analisado está querendo transmitir. Uma verdadeira análise qualitativa necessita de conhecimento de termos que estruturam as pesquisas qualitativas: problematização, elaboração de estratégias, organização, produção de texto que seja fiel aos achados, assegurar validação e fidedignidade.

A escolha do material utilizado para análise teve como critério o fato de pertencer a um site confiável, não ser imagem usada em campanhas antigas e apresentar boa quantidade de textos para serem analisados. Outras imagens encontradas na internet, apesar de interessantes, continham poucas frases para serem analisadas e inúmeras pertenciam a site de blogs. O material escolhido possui uma imagem do site do INCA do ano de 2016 que não seria um ano tão distante do atual.

\subsection{Justificativa}

Segundo Meirelles (2017), o principal fator contribuinte para a mortalidade prematura é o tabagismo, que poderia ser evitável. A Organização Mundial de Saúde - OMS constatou no ano de 2011, que o fumo era o protagonista de cerca de 6 milhões de mortes anualmente. Para 2020, a projeção da mortalidade devido ao uso do tabaco é de 7,5 milhões. Além disso, será computado em sua conta $90 \%$ do câncer de pulmão em homens e $70 \%$ em mulheres e $22 \%$ para doenças relacionadas ao sistema cardiovascular (ALMEIDA; MUSSI, 2006)

Entre 56-80\% para doença pulmonar obstrutiva crônica DPOC se deve a esse hábito. A World Health Organization - WHO estima que a expectativa de vida de quem faz o uso dessa substância nociva se reduza aproximadamente em 15 anos (NUNES; DE CASTRO, 2011).

Além de danos físicos, o tabagismo é responsável por custos sociais e econômicos. Esses custos envolvem a utilização dos recursos da saúde e ausência no trabalho acarretando redução na produtividade. Apesar da venda proibida do tabaco para menores de 18 anos no Brasil, o consumo se inicia cada vez mais cedo sendo a média de idade do começo do uso de 15 anos (NUNES; DE CASTRO, 2011). Isso diminui a expectativa de vida da população ativa, pois muitos desses adolescentes desenvolvem doenças, que evoluem a óbito decorrente da comorbidade por ação desse hábito.

Para que esses jovens tenham mais conhecimento sobre o assunto e visando orientar adultos e idosos, foi criada a lei $n^{\circ} 7488$ de 11 de junho de 1986 que originou o Dia Nacional de Combate ao Fumo, em 29 de agosto. Essa lei tem o intuito de alertar os brasileiros sobre os malefícios do tabagismo. 
Diante disso, é preponderante aproveitar esse dia para a conscientização da população, por meio de palestras, vídeos, fotos catastróficas decorrentes desse hábito, folders, cartazes e propagandas para que se consiga atingir a maior parte da população englobando tanto tabagistas, como os que não fumam (BRASIL, 1986).

Estudos mostram que o tabagismo, na maioria dos casos, se inicia na adolescência por ser esta uma fase em que os jovens são muito influenciados, buscam sua liberdade, novas experiências e desejo pela vida adulta. Frequentemente, os jovens não compreendem a proporção dos malefícios acarretados para sua vida com esse costume, apenas o faz como meio de inserção em uma roda de amigos. Nunes e Castro (2011) afirmam que com a precoce aquisição desse costume é observado dependência mais grave no futuro. Nessa situação e em decorrência da propagação dos malefícios do tabaco entre esses adolescentes é fundamental desenvolver ações de promoção de saúde e prevenção para aqueles que ainda não adquiriram essa prática. Entretanto, para que atinja essa população de adolescentes é importante um mecanismo que os faça se interessar pelo assunto abordado e induzir à prática da abstinência relativa a esse hábito. Os mecanismos utilizados para sensibilização tem sido a comunicação visual.

Echer (2005) defendia a elaboração de manuais para o cuidado da saúde. Estes devem conter uma linguagem que atinja todas as camadas sociais independente do grau de escolaridade. Segundo Freitas e Rezende Filho (2011), essas ferramentas para a educação em saúde como folders, cartilhas e cartazes fortalecem e uniformizam as orientações, facilitando o trabalho da equipe multidisciplinar. Essa ideia vai ao encontro do proposto por Zerwic et al. (2010) que afirmavam a existência de uma apresentação visual eficaz na propagação da mensagem contida nesses materiais educativos.

Quando o assunto abordado diz a respeito à ação contra o tabagismo por meio de campanhas visando a população, é válido o uso de imagens e dados impactantes para o leitor. Para Marques (2018), a dependência química, psicológica e social causada pelo uso do tabaco deve ser o foco dos materiais educativos.

\subsection{Educação em Saúde}

O termo educação em saúde, usado na atualidade, originouse nas primeiras décadas do século XX em que as ações do estado se davam por meio de campanhas sanitárias e estas eram estratégias de educação em saúde de forma autoritária, pois a população era vista como incapaz de tomar iniciativas próprias (FALKENBERG et al., 2014). Atualmente, a situação se modificou. São feitas campanhas para a propagação da informação com o intuito de que a população adquirira conhecimento de uma forma rápida e prática e que comece a ser o agente ativo da mudança de hábitos, ou seja, passou a participar ativamente dessas mudanças.

Com o passar dos anos em que a medicina deixou de ser somente curativa para ser preventiva foi fundamental a educação em saúde, pois essa educação é a formação de saberes na área da saúde para que a população se conscientize. Essa perspectiva concebe a possibilidade de mudança de hábitos que acarretem melhorias na sua saúde e até mesmo da convivência em comunidade. Pode-se ter um exemplo comum no caso da dengue, em que as pessoas são orientadas a não deixarem água parada, cuidar dos reservatórios para que não fiquem abertos. Essas precauções para evitar que o mosquito se propague são levadas para a população por meio de educação na área da saúde, podendo ser de forma oral, com palestras, ou de forma escrita, como cartazes, folders e contribuirão para que haja um estreitamento na relação entre as pessoas. Da mesma forma, pode-se supor que campanhas contra o tabagismo promovam esse estreitamento relacional, além de proporcionar melhorias na saúde pessoal, familiar e coletiva, na economia pessoal e familiar.

Nos dias atuais, como os aparatos tecnológicos estão disponíveis com grande expressividade podem ser um meio de propagação dessa educação para a população sem discriminar faixa etárias e classes sociais. É dessa forma que textos ou cartazes digitalizados entram na conta dos fatores contributivos para a educação em saúde da população em geral e da população jovem, em especial (FREITAS; REZENDE FILHO, 2011).

Para Falkenberg et al. (2014), existem três atores na prática de educação em saúde. Estes seriam os profissionais da área da saúde, prevenindo e promovendo, os gestores com a finalidade de apoiarem os profissionais na execução e a população que diante da educação proporcionada deve construir um conhecimento para que possa aumentar a autonomia nos cuidados, tanto individuais quanto coletivos. Acrescentaríamos a esta relação de Falkenberg os materiais disponibilizados, isto é, a sua forma, o seu conteúdo. Para esta pesquisa foi alisado apenas o conteúdo levando em conta a tipologia de conteúdos proposta por Zabala (1998).

Reberte, Hoga e Gomes (2012, p.2) complementam que a relevância dos materiais educativos impressos ou digitais, como os que são utilizados na Educação em Saúde, “depende dos princípios e das formas de comunicação envolvidos no processo de elaboração".

Normalmente esses materiais pautam-se pela impessoalidade, concisão no texto escrito, cores atrativas e discretas e figuras, humanas ou não, em consonância com o tema da campanha que se pretende implementar. Essas características dos elementos constitutivos são pensadas levando em conta a espécie de público que se pretende atingir e as circunstâncias em que ele se encontra. Além de informar sobre fatos devem ser articulados elementos constitutivos de modo a contribuir para possíveis alterações de atitude.

\subsection{Análise}

A análise do material educativo em pauta levou em conta os tipos de conteúdos veiculados no mesmo. Esses tipos conteúdos revelam a concepção de valor que se atribui à 
educação coletiva, trazem implícitas finalidades da campanha ao responderem à pergunta: o que a sociedade deve saber sobre esse tema?

Uma forma de conceber o processo educativo é considerar que a educação tem por objetivo levar ao aprendizado de fatos, conceitos, procedimentos e induzir a construção de atitudes. Sabe-se que a prática do que se aprende decorre também de outras instâncias, a saber: família, sociedade, igreja, etc. Nessa perspectiva a aprendizagem não é um fenômeno apenas cognitivo e o conhecimento em si tem poder de metabolizar as informações oriundas das outras fontes e transformá-las em atitudes e mobilizar o sujeito da aprendizagem para a ação. Conhecendo os fatos, o sujeito constrói os conceitos e, sabendo das implicações, modificará os seus procedimentos a partir de informações complementadas pelas outras instituições (MIZUKAMI, 1986).

Essas campanhas educativas em saúde concebem o processo educativo como responsável pelo envolvimento do sujeito como um todo. Elas se preocupam com a realização da pessoa. Focalizam as formas humanas de conhecer e se transformar: a cognição, os aspectos sensoriais, a emoção e o aspecto social. Tal educação não se restringe à abordagem dos fatos e conceitos, mas estende-se à orientação de procedimentos e construção de valores visando a constituição de atitudes duradouras (MARTINS; BICUDO, 2006). Dessa forma, destaca-se a importância da elaboração de materiais educativos utilizados em campanhas de saúde com conteúdos que prendam a atenção do leitor, procurem despertar seu interesse e tenha potencial para proporcionar mais do que oportunidade de aprendizagem de conceitos e fatos. Um material que procure sensibilizar para a tomada de decisão, de valorização de vida, do bem-estar e do bem-viver.

Para Zabala (1998) normalmente, os conteúdos que levam à aprendizagem são discriminados de acordo com os objetivos e a perspectiva de educação. Assim, ao se elaborar cartilhas, folders ou cartazes para orientar a população é fundamental a análise desse material para ofertar todos os conteúdos. Salientando que em um conteúdo, mesmo que este seja especifico, pode ter classificações associadas.

Os conteúdos factuais são inquestionáveis, memorizados, e costumam ser postos a prova, conforme Zabala (1998). Diante do esclarecimento, ao fazer conexão com o tema trabalhado aqui, quando se toma frase "O tabagismo acarreta malefícios à saúde", pode-se notar que é um fato epidemiológico e a população, mesmo que não saiba quais danos especificamente são provocados à saúde pelo uso do cigarro, está convencida de que algo mal é produzido pelo seu uso. As pesquisas revelaram e a imprensa, especializada ou não, encarregou-se da ampla divulgação desse fato.

Os conceitos e princípios, por sua vez, são construídos a partir de conjunto de fatos e essa construção é um processo gradual demandando mais ou menos tempo conforme a pessoa, ou o conceito a ser construído. Zabala (1998) os classificou como termos abstratos e que a compreensão para a formação de conceitos é de extrema importância. Por exemplo, na frase. "Cigarro faz mal à saúde", temos um conceito implícito na frase "fazer mal". Discutir o que é "fazer mal" é analisar um conceito e aprofundar a compreensão do seu significado, que varia com o tempo e de acordo com a pessoa e sua compreensão dos fatos. Que o cigarro faz mal à saúde é também um fato e essa informação tem por objetivo impactar a sociedade para que tome atitude de delimitar espaços de uso do tabaco e incluir o tema em suas discussões familiares, educacionais e de planejamento de políticas públicas.

Os conteúdos procedimentais são ações que incluem habilidades e destreza. A fazer a divisão desses conteúdos em segmentos em um deles encontram-se as ações tanto motoras quanto cognitivas podendo incluir verbos como recortar, saltar, ler, traduzir, opinar. Ao lermos a seguinte frase: "Não permita que o cigarro domine sua vida, abandone- o agora mesmo" pode -se observar que não permitir e abandonar o cigarro são conteúdos procedimentais, pois indicam ação resultante de uma atitude.

Os conteúdos atitudinais, conforme o nome indica, são atitudes esperadas, que normalmente não estão explícitas no texto, mas que podem ser percebidas nas figuras e estimuladas pelo contexto geral apresentado no material educativo. $\mathrm{O}$ valor atribuído pode variar de pessoa para pessoa, mas há valores mais amplos tendem a ser cultivados por todos. Diante disso, na frase "não permita que o cigarro domine sua vida" mesmo que não tenha a continuação da frase como no exemplo acima, é evidente que o indivíduo que está lendo associará à orientação de não utilizar mais o cigarro, pois faz mal. E essa seria a atitude esperada, porém para que este possa realizar essa ação, ela deve entender a mensagem e esta serlhe relevante.

Desta forma, ao entender as diferentes tipologias de conteúdos descritas acima, torna-se possível a classificação dos textos ou frases utilizadas em materiais educativos na área educação em saúde (Figura 1).

Figura 1- Cartaz Produzido pelo INCA

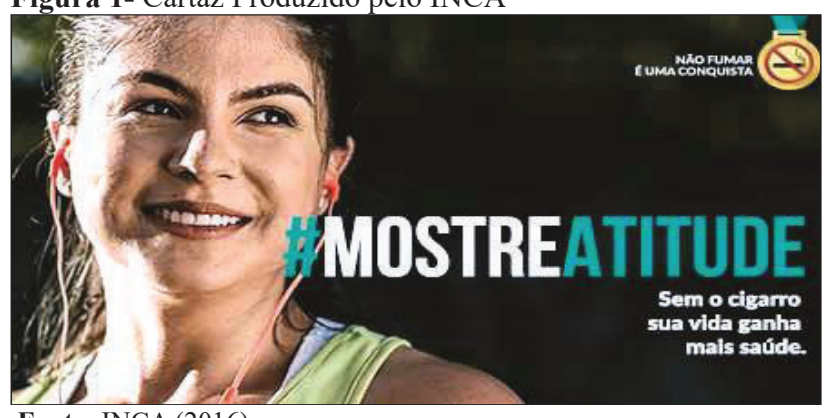

Fonte: INCA (2016).

Para elaborar frases com finalidade de aprendizagem e mudança de atitude em uma população é importante que a mensagem contemple os conteúdos de todos os tipos (Quadro $1)$. 
Quadro 1 - Tipos de conteúdo extraídos da figura

\begin{tabular}{|c|c|c|}
\hline Enunciado & Tipo de Conteúdo & Justificativa \\
\hline Mostre atitude & $\begin{array}{c}\text { Conceitual, } \\
\text { procedimental e } \\
\text { atitudinal }\end{array}$ & $\begin{array}{c}\text { Estão presentes os conceitos de atitude, o procedimento resultante e a própria } \\
\text { atitude. Há, nesse enunciado conciso, um procedimento esperado resultante } \\
\text { da atitude anunciada e, finalmente, a decisão esperada ou atitude propriamente } \\
\text { dita. }\end{array}$ \\
\hline $\begin{array}{c}\text { Não fumar, uma } \\
\text { conquista }\end{array}$ & Conceitual, atitudinal & O conceito de conquista e atitude de não fumar. \\
\hline $\begin{array}{c}\text { Sem o cigarro sua vida } \\
\text { ganha mais saúde }\end{array}$ & Conceitual e factual & $\begin{array}{c}\text { O conceito presente, seria "mais saúde" ou vida com mais saúde. O conteúdo } \\
\text { factual presente consiste no fato de vida ganhar mais saúde. }\end{array}$ \\
\hline
\end{tabular}

Fonte: Dados da pesquisa.

No Quadro 1 foram destacados três enunciados com finalidades educativas. O primeiro é: "mostre atitude". Um imperativo. De acordo com esse excerto da mensagem, atitude é um conceito que neste caso significa um modo de pensar, domínio da vontade e autocontrole seletivo. O desafio proposto, demostrar atitude, tem por finalidade induzir a uma ação, o procedimento de abandonar o cigarro. Por essa razão foi classificado também como um conteúdo atitudinal. Dado que o esperado é que tal atitude produza uma ação é também um conteúdo procedimental. Esta será a resposta que evidenciará a atitude, portanto, nesse enunciado sintético e imperativo estão presentes três tipos de conteúdos.

O segundo enunciado apresenta o conceito de conquista. A conquista de algo subjetivo, de valor intrínseco para o sujeito, que é o autodomínio, a autonomia em relação ao hábito de fumar. Ao mesmo tempo o enunciado induz à atitude de não fumar para viver melhor. Um esforço para subverter a concepção usual de que "viver bem" é como sinônimo de viver como quer, usufruindo momentos de prazer. Determina o conceito de "viver bem" enquanto uma conquista de hábitos saudáveis e possibilidade de vida com minimização de males.

O terceiro enunciado "Sem o cigarro sua vida ganha mais saúde" destaca um fato longamente observado e constatado, pois em conformidade com Inca o cigarro possui 4700 substâncias tóxicas ao organismo, como apresentado anteriormente, o que acarreta doenças que poderiam ser evitáveis com o abandono desse hábito. Além disso, devese ter em mente o conceito de saúde que, segundo a OMS é definido de acordo como a pessoa se apresenta fisicamente, mentalmente e também na sociedade. Se está em um bom estado físico, mental e social pode-se dizer que o indivíduo estará saudável (SEGRE; FERRAZ, 1997).

Portanto, o autocontrole, o abandono do hábito de fumar, a preocupação com o bem-estar físico e social, são indicativos de saúde.

\section{Conclusão}

Nos dados apresentados sobre os malefícios causados à saúde, pelo uso do tabaco, ficou evidente a necessidade orientar a sociedade sobre o inconveniente desse hábito. Observa-se que muitos jovens, adultos e idosos, parecem não ter se despertado para a necessidade de se proteger contra esses males e a Educação em Saúde tem recorrido a diversos recursos midiáticos contendo uma linguagem acessível, e pretensamente impactante, com cores e imagens que possam despertar o olhar para frases que contenham conteúdos educativos e que tenham potencial para causar impacto, induzir a uma atitude de mudança desse hábito.

Uma imagem escolhida tem uma mensagem, escrita, curta, mas rica em conteúdo. Tanto é assim que contempla os quatro tipos de conteúdos de ensino de que tratou Zabala.

Observou-se que no que diz respeito às campanhas contra o tabagismo o material é sintético, uma vez que de longo tempo se anuncia os malefícios do cigarro e outros produtos equivalentes, restando agora a tentativa de causar impacto no fumante através de frases de efeito.

Desta forma, ficou evidente que os educadores em saúde entenderam a necessidade de não mais destacar fatos científicos, mas apelar para uma mudança de atitude. Resta a necessidade de analisar os efeitos práticos da campanha sobre as pessoas.

\section{Referências}

ALMEIDA, A. F.; MUSSI, F.C. Tabagismo: conhecimentos, atitudes, hábitos e grau de dependência de jovens fumantes em Salvador. Relato de pesquisa. Rev. Esc. Enferm., 2006. doi: http://dx.doi.org/10.1590/S0080-62342006000400002

BRASIL. LEI No 7.488, DE 11 DE JUNHO DE 1986. Institui o Dia Nacional de Combate ao Fumo. 1986. Disponível em: $<$ http://www.planalto.gov.br/ccivil_03/leis/1980-1988/L7488. htm> Acesso em: 13 nov. 2019.

BRASIL. Ministério da Justiça. Secretaria Nacional de Políticas sobre Drogas. Drogas: cartilha sobre tabaco. Brasília: Ministério da Justiça, 2011.

CELLARD, A. A análise documental. In: POUPART, J. et al. $A$ pesquisa qualitativa: enfoques epistemológicos e metodológicos. Petrópolis: Vozes, 2008. p.295-298

ECHER, I.C. Elaboração de manuais de orientação para o cuidado em saúde. Rev. Latinoam. Enferm., v.13, n.5, p,754-757, 2005.

FALKENBERG, M.B. et al. Educação em saúde e educação na saúde: conceitos e implicações para a saúde coletiva. Ciênc. Saúde Coletiva, v.19, n.3, p.847-852, 2014.

FREITAS F.V., REZENDE FILHO L.A. Modelos de Comunicação e uso de impressos na educação em saúde: uma pesquisa bibliográfica. Interface Com. Saúde Educ., v.15, n.36, p.243-55, 2011. doi: http://dx.doi.org/10.1590/S141432832010005000044 . 
INCA. Instituto Nacional de Câncer. O controle do tabaco no Brasil: uma trajetória. 2016. Disponível em: $<$ https://www.inca. gov.br/exposicoes/o-controle-tabaco-brasil-uma-trajetoria>. Acesso em: 25 jan. 2020.

INCA - Instituto Nacional do Câncer. Dia Nacional de Combate ao Fumo - 2016. Disponível em: <www.inca.gov.br/campanhas/ dia-nacional-de-combate-ao-fumo/2016/mostre-atitude-sem-ocigarro-sua-vida-ganha-mais-saude> Acesso em: 17 set. 2019.

MARQUES, A.C.P.R. et al. Consenso sobre o tratamento da dependência de nicotina. Departamento de Dependência Química da Associação Brasileira de Psiquiatria. Rev. Bras. Psiquiatr., v.23, n.4, p.200-214, 2001

MARQUES, F.A. Análise de um material educativo sobre tabagismo a partir do referencial teórico da tipologia de conteúdos. In: SALES, A.; CERCHIARI, E.A.N. (Org.). Educação em Saúde: Análise de conteúdos de ensino- aprendizagem em materiais. Campo Grande: Life, 2018, p.58-69.

MARTINS, H.H.T.S. Metodologia qualitativa de pesquisa. Educ. Pesq., v.30, n.2, p. 289-300, 2004.

MARTINS, J.; BICUDO, M.A.V. Estudos sobre o existencialismo, fenomenologia e educação. São Paulo: Centauro, 2006.

MEIRELLES, K. Dia Nacional de Combate ao Fumo: tabagismo mata 6 milhões de pessoas por ano no mundo. Agência $I B G E$ Notícias, 2017. Disponível em: https://agenciadenoticias.ibge. gov.br/agencia-noticias/2012-agencia-de noticias/noticias/16075pesquisas-do-ibge-auxiliam-politicas-de-prevencao-do-uso-detabaco>. Acesso em: 15 jan. 2020.
MINAYO, M.C.S. Análise qualitativa: teoria, passos e fidedignidade. Ciênc. Saúde Coletiva, v.17, n.3, p.621-626, 2012.

MIZUKAMI, M.G.N. Ensino: as abordagens do processo. São Paulo: EPU, 1986.

NUNES, S.O.V.; DE CASTRO, M.R.P. Tabagismo: abordagem, prevenção e tratamento. Londrina: EDUEL, 2011.

OMS - Organização Mundial da Saúde. Cuidados de Saúde Primários - Agora Mais Que Nunca. Relatório Mundial de Saúde 2008. 2011. Disponível em: <https://www.who.int/eportuguese/ publications/pt/> Acesso em: 25 out. 2019.

REBERTE, L. M.; HOGA, L. A. K.; GOMES, A. L. Z. O processo de construção de material educativo para a promoção da saúde da gestante. Rev. Latinoam. Enferm., v.20, n.1, p.101-108, 2012. doi: https://doi.org/10.1590/S0104-11692012000100014.

SALEHEEN, D.; ZHAO, W; RASHEED, A. Epidemiology and public health policy of tobacco use and cardiovascular disorders in low- and middle-income countries. Arterioscler Thromb. Vasc. Biol., v.34, n.9, p.1811-1819, 2014. doi: 10.1161/ ATVBAHA.114.303826.

SEGRE, M.; FERRAZ, F.C. O conceito de saúde. Rev. Saúde Pública, v.31, n.5, p.538-542, 1997.

ZABALA, A. A prática educativa: como ensinar. Porto Alegre: Artmed, 1998.

ZERWIC, J.J. et al. Tips for better visual elements in posters and podium. Educ. Health Change Learning Practice, v.23, n.2, p.267, 2010. 